\title{
Boltzmann equation approach for transport in systems subject to microwave irradiation
}

\author{
Tobias Brandes \\ Department of Physics, Gakushuin University, 1-5-1 Mejiro, Toshima-ku, Tokyo 171, Japan
}

\begin{abstract}
We calculate the electronic transport properties of a system which is irradiated by a homogeneous microwave field. Within a Boltzmann equation approach, a general expression for the conductivity tensor is derived and evaluated for a quasi one-dimensional ballistic quantum wire and a two dimensional system with impurity scattering. For the latter, deviations from the Drude result are predicted for the conductivity tensor. This should be observable in systems where the scattering rate depends noticeably on the Fermi energy. The deviations are calculated explicitly for the case of a $2 \mathrm{~d}$ Silicon layer, where they strongly depend on the microwave polarization and the Fermi energy.
\end{abstract}

PACS: $72.10 \mathrm{Bg}$ (General formulation of transport theory), 72.15 Lh (Relaxation times and mean free paths), $72.30+q$ (High frequency effects; plasma effects)

\section{INTRODUCTION}

Recently, frequency dependent effects have been discussed for the transport properties of a large variety of systems. Examples are periodically driven quantum wells [1], photonassisted tunneling through double barrier structures [2, 3], and photo-induced transport through quantum point contacts [ []]. In a two-dimensional system, the quantum Hall effect has been investigated experimentally in the presence of an additional microwave field [5.6].

A common feature of these investigations is that the external frequency dependent field (which we call 'microwave' in the following) is an intrinsic part of the system itself. This means that it does not act as a 'probe field' to which the response is tested as in an ordinary AC transport experiment. Rather, one has to think of transport in a DC 'probe' field in presence of an additional, time dependent field that eventually changes the DC transport properties as do other perturbations like, e.g., the electron-electron or electron-phonon interaction. From the theoretical point of view, one therefore has to reformulate the DC response theory. In a quantum mechanical description, one would include the microwave into the Hamiltonian which then becomes time dependent.

In this work, we use a different approach and derive the electronic transport, namely the conductivity, in the semiclassical regime, where a description in terms of the Boltzmann equation is expected to be valid. Naturally, phenomena like localization, strong correlation effects or the mesoscopic regime are beyond the validity of such a theory. As we show below, however, even in the classical regime nontrivial effects can arise in the DC transport due to the influence of an additional, time-dependent perturbation. Moreover, while the transport in the linear regime always has to be understood in the limit of a vanishing DC 'probe 
field', the microwave field still could be arbitrarily strong and new, nonlinear effects can be expected.

In the following, we derive a general expression for the current linear in an external 'probe field' in presence of an additional microwave within the relaxation time approximation. As an example, we first calculate the conductance of a clean quantum wire, where the influence of the microwave, however, vanishes in the DC limit. This is not the case for the second example of a two dimensional silicon accumulation layer. There, the impurity scattering causes a scattering rate that strongly depends on the electron's quasi momentum. The latter is changed periodically due to the microwave which in turn eventually gives a deviation of the conductivity from the standard Drude result. We calculate this deviation to second order in the microwave and find it to be negative for most values of the Fermi energy. Furthermore, it depends on the microwave polarization and should be detectable in an ordinary DC measurement of the 2 d layer under microwave irradiation.

\section{BOLTZMANN EQUATION}

The central concept for the description of electronic transport properties in the framework of the Boltzmann equation is the semi-classical distribution function $f(\mathbf{r}, \mathbf{k}, t)$ in phase space. Scattering of electron wave packets at impurities, phonons or other electrons is governed by the equation (we set $\hbar=1$ )

$$
\left(\partial_{t}+\mathbf{v}_{\mathbf{k}} \partial_{\mathbf{r}}+\mathbf{F}(\mathbf{r}, t) \partial_{\mathbf{k}}\right) f(\mathbf{r}, \mathbf{k}, t)=-\mathcal{L} f(\mathbf{r}, \mathbf{k}, t),
$$

where $\mathbf{F}(\mathbf{r}, t)$ is the force exerted on the electrons by an arbitrary electric field and $\mathbf{v}_{\mathbf{k}}=\mathbf{k} / \mathrm{m}$ the velocity of electrons with bandmass $m$ and quasi momentum $\mathbf{k}$. Here and in the following we do not include a magnetic field. The collision term $-\mathcal{L} f(\mathbf{r}, \mathbf{k}, t)$ is a nonlinear operator acting on the distribution function $f$ and describes its change due to scattering depending on the microscopic scattering mechanism. Within the quasiclassical description of linear response quantities, we are able to include exactly the influence of an arbitrary strong, spatially homogeneous microwave field.

The first step is to distinguish in Eq. (1) between two different contributions to $\mathbf{F}(\mathbf{r}, t)$ : First, the force $e \mathbf{E}_{0}(t)$ exerted on the electron by the microwave ( $e$ denotes the electron charge). Second, the 'probe field' $\mathbf{E}(\mathbf{r}, t)$ which is generated by driving a current through the system. The proportionality between the latter and the probe field is used to define linear response quantities like the conductivity. Using this decomposition $\mathbf{F}(\mathbf{r}, t)=e \mathbf{E}_{0}(t)+$ $e \mathbf{E}(\mathbf{r}, t)$, the linear response to the probe field can be calculated.

We now solve the Boltzmann equation to linear order in the probe field $\mathbf{E}(\mathbf{r}, t)$. The distribution function is decomposed into

$$
f(\mathbf{r}, \mathbf{k}, t)=f_{0}(\mathbf{k}(t))+\delta f(\mathbf{r}, \mathbf{k}, t),
$$

where $f_{0}(\mathbf{k}(t))$ is the solution in absence of the probe field,

$$
f(\mathbf{r}, \mathbf{k}, t)=f_{0}(\mathbf{k}(t)), \quad \mathbf{k}(t)=\mathbf{k}-e \int_{0}^{t} d t^{\prime} \mathbf{E}_{0}\left(t^{\prime}\right)=\mathbf{k}+\frac{e}{c} \mathbf{A}^{e}(t)
$$

where $\mathbf{A}^{e}(t)$ is the vector potential of the microwave field with $\mathbf{E}_{0}(t)=-\partial \mathbf{A}^{e}(t) / \partial(c t)$. (Note that the Fermi distribution function $f_{0}(\mathbf{k})=f(\mathbf{r}, \mathbf{k}, t=0)$ gives zero upon inserting 
into the collision term). We obtain an equation for the change $\delta f(\mathbf{r}, \mathbf{k}, t)$ due to the probe field

$$
\left(\partial_{t}+\mathbf{v}_{\mathbf{k}} \partial_{\mathbf{r}}+e \mathbf{E}_{0}(t) \partial_{\mathbf{k}}\right) \delta f(\mathbf{r}, \mathbf{k}, t)+e \mathbf{E}(\mathbf{r}, t) \partial_{\mathbf{k}} f_{0}(\mathbf{k}(t))=-\mathcal{L}\left[f_{0}(\mathbf{k}(t))+\delta f(\mathbf{r}, \mathbf{k}, t)\right] .
$$

Note that in Eq. (田) the microwave field $\mathbf{E}_{0}(t)$ is still fully included whereas the probe field $\mathbf{E}(\mathbf{r}, t)$ is considered only to lowest order.

In general, since the Boltzmann equation is a nonlinear integral equation, it can not be solved exactly. In the following, we restrict ourselves to the relaxation time approximation, $\mathcal{L} \delta f(\mathbf{r}, \mathbf{k}, t)=\tau^{-1}(\mathbf{k}) \delta f(\mathbf{r}, \mathbf{k}, t)$, where $\tau^{-1}(\mathbf{k})$ is the scattering rate for electrons with quasi momentum $\mathbf{k}$. In the case of elastic impurity scattering, $\tau^{-1}(\mathbf{k})=1 / L^{d} \sum_{\mathbf{k}^{\prime}} W_{\mathbf{k k}^{\prime}}$, where $W_{\mathbf{k k}^{\prime}}=2 \pi n_{i} \delta\left(\varepsilon_{k}-\varepsilon_{k^{\prime}}\right)\left|\left\langle\mathbf{k}|V| \mathbf{k}^{\prime}\right\rangle\right|^{2}$ and $V$ is the potential of impurities with a concentration $n_{i}$ in a system of volume $L^{d}$. In the limit of weak fields $\mathbf{F}(\mathbf{r}, t) \rightarrow 0$, the relaxation time approximation becomes exact for isotropic potentials such that $W_{\mathbf{k k}^{\prime}}=W_{|\mathbf{k}|\left|\mathbf{k}^{\prime}\right|}$. In general, this approximation replaces the effect of the collision term by a rate $\tau^{-1}(\mathbf{k})$ describing the relaxation of a deviation $\delta f$ of the distribution function towards its quasi-equilibrium value $f_{0}(\mathbf{k}(t))$, Eq. (3) due to scattering.

It is a standard procedure to solve the resulting first order partial differential equation. The solution can be obtained by the methods of characteristics [0] and reads

$$
\begin{aligned}
\delta f(\mathbf{q}, \mathbf{k}, t) & =-\partial_{\mathbf{k}} f_{0}(\mathbf{k}(t)) \int_{0}^{t} d t^{\prime} e \mathbf{E}\left(\mathbf{q}, t^{\prime}\right) \exp \left\{-\int_{t^{\prime}}^{t} d s g\left[\mathbf{k}(t)+e \int_{0}^{s} d t^{\prime \prime} \mathbf{E}_{0}\left(t^{\prime \prime}\right)\right]\right\} \\
g(\mathbf{k}) & :=\tau^{-1}(\mathbf{k})+i \mathbf{v}_{\mathbf{k}} \mathbf{q}
\end{aligned}
$$

where we Fourier transformed $\mathbf{r} \rightarrow \mathbf{q}$. The current density is obtained from the resulting change $\delta f(\mathbf{q}, \mathbf{k}, t)$ of the distribution function as

$$
\begin{aligned}
\mathbf{j}(\mathbf{q}, t) & \equiv \frac{e}{L^{d}} \sum_{\mathbf{k}} \mathbf{v}_{\mathbf{k}} f(\mathbf{q}, \mathbf{k}, t)=\frac{e^{2}}{L^{d} m} \sum_{\mathbf{k}}\left[\mathbf{k}-\frac{e}{c} \mathbf{A}^{e}(t)\right]\left(-\partial_{\mathbf{k}} f_{0}(\mathbf{k})\right) \times \\
& \times \int_{0}^{t} d t^{\prime} \mathbf{E}\left(\mathbf{q}, t^{\prime}\right) \exp \left\{-\int_{t^{\prime}}^{t} d s g\left[\mathbf{k}+e \int_{0}^{s} d t^{\prime \prime} \mathbf{E}_{0}\left(t^{\prime \prime}\right)\right]\right\}-\frac{e^{2}}{m c} n_{e} \mathbf{A}^{e}(t) .
\end{aligned}
$$

Here, we shifted the $\mathbf{k}$-summation according to $\mathbf{k} \rightarrow \mathbf{k}-(e / c) \mathbf{A}^{e}(t)$ in Eq. (6), and $n_{e}$ is the electronic density.

Eq. (6) is the central result, expressing the response of the current to the probe field, and valid within the relaxation time approximation for arbitrary microwave field strength $\mathbf{E}_{\mathbf{0}}$. The scattering rate $\tau^{-1}(\mathbf{k})$, however, in general has a nontrivial dependence on $\mathbf{k}$ which makes it impossible to evaluate Eq. (6) analytically.

The conductivity $\sigma(\mathbf{q}, z)$ is defined by the component of the current density $\mathbf{j}_{p r}(\mathbf{q}, t)$ with a time dependence proportional to $\exp (-i z t)$, the time dependence of the probe field:

$$
\mathbf{j}_{p r}(\mathbf{q}, t)=\sigma(\mathbf{q}, z) \times \mathbf{E}(\mathbf{q}) \exp (-i z t)
$$

In the case of a constant scattering rate $\tau^{-1}(\mathbf{k})=\tau^{-1}$, the $t^{\prime}$ integration in Eq. (6) can be performed and one obtains an analytical expression for the conductivity Eq. (7). We evaluate its real part $\sigma^{\prime}$ for a quasi one dimensional wire, where it serves as a definition of the conductance [8,9]. It can be evaluated for the ballistic case, which is defined by the limit 
of vanishing scattering rate, $\tau^{-1} \rightarrow 0$, describing a 'clean' quantum wire in the limit of only one occupied subband in absence of any scattering. The result is

$$
\sigma^{\prime}(q, \omega)=\sum_{n=-\infty}^{\infty} J_{n}^{2}\left(\frac{e E_{0} q}{m \Omega^{2}}\right) \sigma_{0}^{\prime}(q, \omega-n \Omega) \frac{\omega}{\omega-n \Omega},
$$

where $\sigma_{0}^{\prime}(q, \omega)=\left(e^{2} / L\right) \sum_{k} v_{k}\left(-\partial_{k} f_{0}(k)\right) \pi \delta\left(\omega-v_{k} q\right)$ is the real part of the conductivity in absence of the microwave. This results coincides with a quantum mechanical calculation 10 for $\hbar \Omega \ll \varepsilon_{F}\left(\varepsilon_{F}\right.$ Fermi energy); the appearance of the Bessel functions is similar to the Tien-Gordon theory [11] of photo-assisted tunneling in superconducting junctions. Thus, we have expressed the conductivity in presence of the microwave in terms of the conductivity $\sigma_{0}^{\prime}(q, \omega)$ in absence of the microwave. The term $n=0$ in Eq. (8) just yields $\sigma_{0}^{\prime}(q, \omega)$, while the terms $n \neq 0$ describe the influence of the microwave. The latter, however, vanishes in the limit of zero (probe field) frequency $\omega \rightarrow 0$. In particular, this means that the $D C$ conductance of a clean quantum wire is not changed under the influence of a homogeneous microwave field [10]. A non-vanishing effect can be expected only in presence of an additional scattering mechanism like impurities or a potential barrier.

\section{TWO DIMENSIONAL SYSTEM}

As a further application of our formalism, we evaluated the conductivity tensor $\sigma(\mathbf{q}=$ 0, z) for a two-dimensional system from Eq. (7) and Eq. (6) to second order in the microwave field $\mathbf{E}_{\mathbf{0}}$. The electron gas and the microwave polarization $\mathbf{E}_{0}=E_{0} \mathbf{e}_{x}$ are assumed to lie in the $x$-y plane. Here, we give the zero-temperature result which holds for $k_{B} T \ll \varepsilon_{F}$, although corresponding expressions for $T>0$ can easily be derived. We obtain

$$
\begin{aligned}
\sigma_{x x} & =: \sigma_{0}+\Delta \sigma_{x x}=\sigma_{0}\left\{1+\frac{\left(\Omega \tau\left(\varepsilon_{F}\right)\right)^{2}}{1+\left(\Omega \tau\left(\varepsilon_{F}\right)\right)^{2}}\left[\frac{3}{8}\left(\frac{v_{F} e E_{0}}{\Omega^{2}}\left(\tau^{-1}\right)^{\prime}\left(\varepsilon_{F}\right)\right)^{2}-\frac{e^{2} E_{0}^{2}}{2 m \Omega^{4} \tau\left(\varepsilon_{F}\right)}\left(\tau^{-1}\right)^{\prime}\left(\varepsilon_{F}\right)\right]\right. \\
& \left.-\left(\frac{e E_{0}}{\Omega}\right)^{2} \frac{\tau\left(\varepsilon_{F}\right)}{4 m}\left[\left(\tau^{-1}\right)^{\prime}\left(\varepsilon_{F}\right)+\frac{3}{2} \varepsilon_{F}\left(\tau^{-1}\right)^{\prime \prime}\left(\varepsilon_{F}\right)\right]\right\}+O\left(E_{0}\right)^{4} \\
\sigma_{y y} & =: \sigma_{0}+\Delta \sigma_{y y}=\sigma_{0}\left\{1+\frac{\left(\Omega \tau\left(\varepsilon_{F}\right)\right)^{2}}{1+\left(\Omega \tau\left(\varepsilon_{F}\right)\right)^{2}} \frac{1}{8}\left(\frac{v_{F} e E_{0}}{\Omega^{2}}\left(\tau^{-1}\right)^{\prime}\left(\varepsilon_{F}\right)\right)^{2}\right. \\
& \left.-\left(\frac{e E_{0}}{\Omega}\right)^{2} \frac{\tau\left(\varepsilon_{F}\right)}{4 m}\left[\left(\tau^{-1}\right)^{\prime}\left(\varepsilon_{F}\right)+\frac{1}{2} \varepsilon_{F}\left(\tau^{-1}\right)^{\prime \prime}\left(\varepsilon_{F}\right)\right]\right\}+O\left(E_{0}\right)^{4}
\end{aligned}
$$

and $\sigma_{x y}=\sigma_{y x}=0$. Here, $\sigma_{0}=e^{2} n_{s} \tau\left(\varepsilon_{F}\right) / m$ is the zero frequency (Drude) conductivity in absence of the microwave ( $m$ denotes the electron band mass). Within the relaxation time approximation, Eq. (9) is valid for both elastic an inelastic scattering, described by the rate $\tau\left(\varepsilon_{F}\right)^{-1}$. Due to the appearance of its derivatives in Eq. (9), one needs to know the dependence of $\tau^{-1}$ on the Fermi energy $\varepsilon_{F}$.

Indeed, in realistic systems this dependence can be quite strong. Years ago, Fang, Fowler and Hartstein [12] measured the Shubnikov-de-Haas oscillations in a Si inversion layer as a function of the gate voltage. The general shape of the magnetoconductivity curve was determined by a broad maximum when plotted against the Fermi energy (compare Fig. 国). This feature was independent of the magnetic field $B$ and could, beside the $B$-dependent 
oscillations, be reproduced in a theoretical calculation by Isihara and Smrčka [13]. They used a pseudopotential model for impurity scattering and a simple CPA approach corresponding to the relaxation time approximation in the Boltzmann equation. The key point was the functional dependence of $\tau(\varepsilon)$, the scattering time, on the energy, namely

$$
\tau(\varepsilon)=a\left[\frac{\varepsilon_{\sigma} \varepsilon}{\varepsilon_{\sigma} \varepsilon^{2}+\varepsilon\left(\varepsilon_{\sigma}^{2}-3 \varepsilon_{\tau}^{2}\right)+\varepsilon_{\sigma} \varepsilon_{\tau}^{2}}\right]^{2},
$$

in lowest order in the impurity concentration. Parameters fitting the experiment [12] where obtained as $a=4.3 * 10^{-10} \mathrm{meV}^{2} \mathrm{~s}, \varepsilon_{\tau}=3.5 \mathrm{meV}$ and $\varepsilon_{\sigma}=15 \mathrm{meV}$. We used Eq. (10) as an example for the evaluation of Eq. (9). The result is shown in the Fig. (1).

An important feature is that under the influence of the microwave the conductivity tensor becomes non-isotropic, i.e. $\sigma_{x x}$ and $\sigma_{y y}$ are different for $E_{0} \neq 0$ (remember that the microwave is assumed to be polarized in $x$-direction). Moreover, the signs of the change $\Delta \sigma_{x x}$ and $\Delta \sigma_{y y}$ due to the microwave depend on the value of the Fermi energy $\varepsilon_{F}$. Indeed, the different terms in Eq. (9) involving both first and second derivative of $\tau^{-1}\left(\varepsilon_{F}\right)$ can even cancel for a certain value of $\varepsilon_{F}$ as is seen in the curve for $\Delta \sigma_{y y}$.

All these features should be detectable in an experiment. For direct comparison with the theoretical result, it would be preferable to work with linearly polarized microwaves with wavelengths longer than any relevant microscopic length scale in the system so that the microwave can be treated in the $\mathbf{q}=0$-limit. Furthermore, other materials then silicon could be used as well, in this case the model for the scattering time Eq. (10) of course has to be modified. However, as long as there is a noticeable dependence of the scattering rate on the energy, the microwave will change the conductance according to Eq. (9). On the other hand, one could imagine a system where the dependence of the scattering rate on the Fermi energy is unknown and not as easily experimentally detectable as was the case in [12]. Then, the microwave could serve as a probe to extract this dependence via Eq. (9).

Since we calculated only to second order in $E_{0}$, the applicability of Eq. (9) is restricted to not too high microwave field strengths. Indeed, the numerical evaluation Fig. (11) shows that at $E_{0} \approx 1000 \mathrm{~V} / \mathrm{m}$ the change $\Delta \sigma_{x x}, \Delta \sigma_{y y}$ becomes of the order of the conductivity $\sigma_{0}$ itself, indicating that above this value of $E_{0}$ one has to go at least to fourth order perturbation theory in the microwave. Furthermore, we did not consider effects like electron heating, ionization or Zener breakdown due to the microwave field but purely the interplay between the momentum conserving impurity scattering and the kinematics of electrons in an oscillating field.

As a summary, we presented a theoretical approach to linear transport in the presence of microwave irradiation within a semiclassical description. The general result for the conductivity was discussed for the case of a two- dimensional Silicon inversion layer, and deviations from the Drude result were predicted to be observable in the zero magnetic field case.

The author would like to acknowledge discussions with A. Kawabata, B. Kramer, G. Platero, R. Kilian and V. Reinstorf, and financial support by the EU STF9 fellowship program in Japan. 
[1] M. Wagner, Phys. Rev. B 49, 16544 (1994).

[2] W. Cai and T. F. Zheng, P. Hu and M. Lax and K. Shum, and R. Alfano, Phys. Rev. Lett. 65, 104 (1990).

[3] J. Iñarrea, G. Platero, and C. Tejedor, Phys. Rev. B 50, 4581 (1994).

[4] S. Feng and Q. Hu, Phys. Rev. B 48, 5354 (1993).

[5] R. Meisels, F. Kuchar, J. J. Harris, and C. T. Foxon, Surface Science 263, 76 (1992).

[6] G. M. Sundaram et al., in ICTP-NATO Workshop on Quantum Dynamics, Vol. 291 of Nato ASI Series, edited by H. Cerdeira, B. Kramer and G. Schön (Kluwer Academic Publishers, Dordrecht/Boston/London, 1994), p. 371.

[7] R. Courant and D. Hilbert, Methods of Mathematical Physics, 2 ed. (Interscience publishers, New York, 1962), Vol. 2.

[8] D. S. Fisher and P. A. Lee, Phys. Rev. B 23, 6851 (1981).

[9] B. Kramer and J. Mašek, Z. Phys. B 76, 457 (1989).

[10] T. Brandes, B. Kramer, and G. Platero, unpublished (1995).

[11] P. K. Tien and J. R. Gordon, Phys. Rev. 129, 647 (1963).

[12] F. F. Fang, A. B. Fowler, and A. Hartstein, Phys. Rev. B 16, 4446 (1977).

[13] A. Isihara and L. Smrčka, J. Phys. C 19, 6777 (1986).

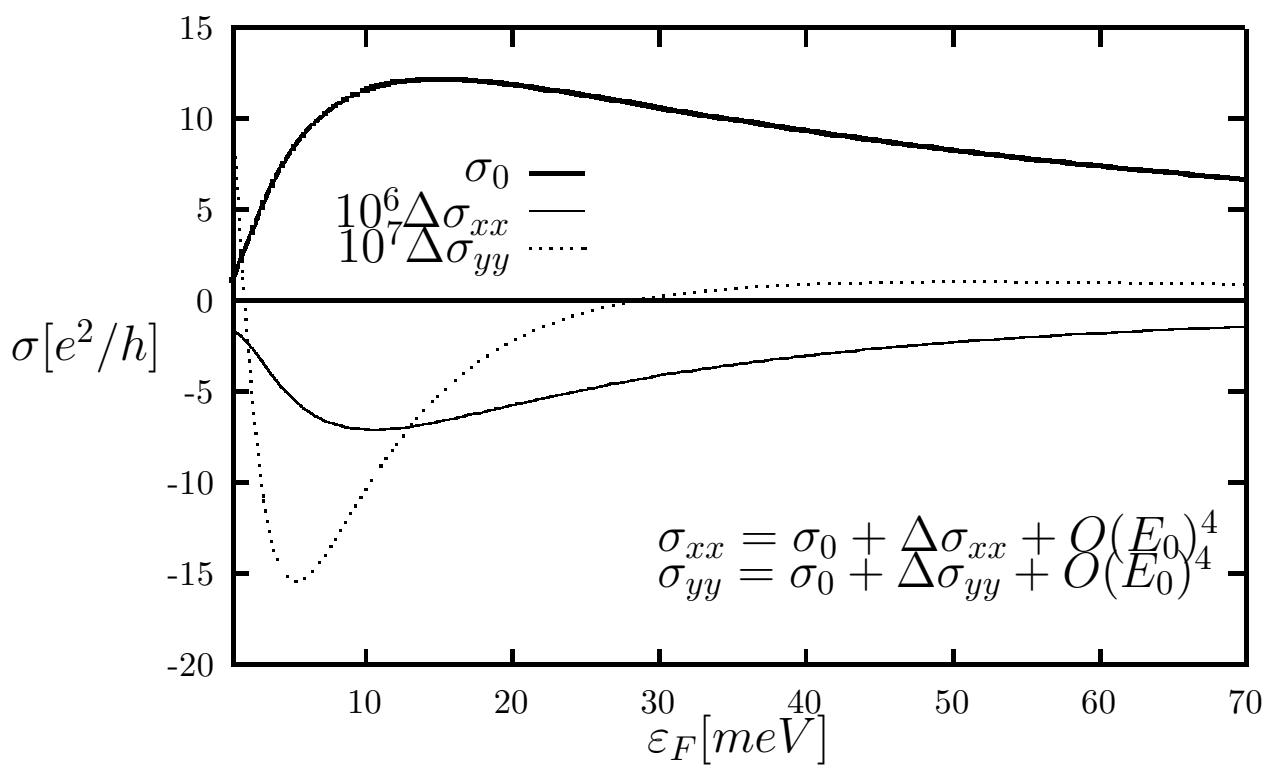

FIG. 1. The calculated zero-temperature conductivity $\sigma_{0}$ (upper curve) as a function of the Fermi energy $\varepsilon_{F}$ in a 2 d Silicon accumulation layer. The scattering of electrons is due to impurities. A microwave field $E_{0}$ (linearly polarized in $x$-direction) with frequency $\Omega=10^{10} s^{-1}$ gives rise to changes $\Delta \sigma_{x x}$ and $\Delta \sigma_{y y}$ of the conductivity (note the different scales), calculated to second order in $E_{0}$ according to Eq. (9). Parameters for the relaxation time as a function of $\varepsilon_{F}$ due to impurity scattering were chosen according to [13]. Curves are shown for $E_{0}=1 \mathrm{Vm}^{-1}$ and have to be scaled with $E_{0}^{2}$ for other values of the microwave field. 